\title{
Probing high-energy acceleration processes in S5 0716+714 using combined Fermi-LAT and MAGIC observations
}

\author{
Marina Manganaro \\ Inst. de Astrofísica de Canarias, E-38200 La Laguna, Tenerife, Spain \\ E-mail: manganaro.marina@gmail.com
}

Bindu Rani*

NASA Goddard Space Flight Center, Greenbelt, MD 20771, USA

E-mail: bindu.rani@nasa.gov

\author{
Giovanna Pedaletti \\ Deutsches Elektronen-Synchrotron (DESY), D-15738 Zeuthen, Germany \\ E-mail: giovanna.pedaletti@desy.de
}

\section{Elina Lindfors}

Finnish MAGIC Consortium, Tuorla Observatory, University of Turku and Astronomy Division,

University of Oulu, Finland

E-mail: elilineutu.fi

\section{for the Fermi-LAT and MAGIC collaborations}

The BL Lac object S5 0716+714 underwent an impressive outburst in January/February 2015, being detected by many instruments spanning from radio to very high energy gamma-rays (VHE, E $>100 \mathrm{GeV}$ ). The multi-wavelength dataset collected made possible a deep study of the broadband Spectral Energy Distribution (SED), which in blazars is described by two components. The low-energy component, in case of S5 $0716+714$ peaked between $10^{14}-10^{15} \mathrm{~Hz}$, is generated by synchrotron emission from relativistic electrons in the jet, while the high-energy one is in general attributed to inverse-Compton (IC) up-scattering of soft synchrotron photons off the same electrons that have undergone synchrotron cooling. For the first time for this source, the high energy ( $\mathrm{HE}, 0.1 \mathrm{GeV}<\mathrm{E}<100 \mathrm{GeV}$ ) and VHE gamma-ray data have been collected simultaneously, from Fermi-LAT and MAGIC respectively, allowing the possibility to study the whole high energy part of the broadband SED in an unprecedented high state. In this contribution we will report on the broadband activity of the source during its strongest outburst ever detected so far, focusing on the GeV-TeV connection, described by the multi-wavelength light curves and the SED. A combined spectrum of Fermi-LAT and MAGIC will be presented, in the frame of a two-zone modelling.

7th Fermi Symposium 2017

15-20 October 2017

Garmisch-Partenkirchen, Germany

\footnotetext{
* Speaker.
} 